\title{
ON THE PRODUCT OF DISTANCES TO A POINT SET ON A SPHERE
}

\author{
GEROLD WAGNER
}

(Received 14 January 1988; revised 17 May 1988)

Communicated by J. H. Loxton

\begin{abstract}
Let $S$ be the surface of the unit sphere in three-dimensional euclidean space, and let $\omega_{N}=$ $\left(x_{1}, x_{2}, \ldots, x_{N}\right)$ be an $N$-tuple of points on $S$. We consider the product of mutual distances $\rho\left(\omega_{N}\right)=\prod_{j \neq k}\left|x_{j}-x_{k}\right|$ and, for the variable point $x$ on $S$, the product of distances $p\left(x, \omega_{N}\right)=$ $\prod_{j=1}^{N}\left|x-x_{j}\right|$ from $x$ to the points of $\omega_{N}$. We obtain essentially best possible bounds for $\max _{\omega_{N}} \rho\left(\omega_{N}\right)$ and for $\min _{\omega_{N}} \max _{x \in S} p\left(x, \omega_{N}\right)$.

1980 Mathematics subject classification (Amer. Math. Soc.) (1985 Revision): 11 K 38, 52 A 40.
\end{abstract}

\section{Introduction}

Let $S$ be the surface of the unit sphere in three-dimensional euclidean space. Given an $N$-tuple of points $\omega_{N}=\left(x_{1}, x_{2}, \ldots, x_{N}\right)$ on $S$, we define a function $p_{N}(x)=p\left(x, \omega_{N}\right)$ to be the product of the euclidean distances from the variable point $x \in S$ to the points $x_{1}, x_{2}, \ldots, x_{N}$ :

$$
p_{N}(x)=p\left(x, \omega_{N}\right)=\prod_{j=1}^{N}\left|x-x_{j}\right| .
$$

What can be said about the maximum value attained by $p_{N}(x)$ on the surface $S$ ? Instead of (1), consider the function

$$
q_{N}(x)=q\left(x, \omega_{N}\right)=\sum_{j=1}^{N}\left(\log \left|x-x_{j}\right|+\log \frac{\sqrt{e}}{2}\right) .
$$

(C) 1989 Australian Mathematical Society $0263-6115 / 89 \$ A 2.00+0.00$ 
It is easy to verify that the integral of $q_{N}(x)$ over the surface $S$ (with respect to the usual surface measure $\sigma$ ) vanishes. This means for the original function $p_{N}(x)$ that the following inequality holds trivially: $\max _{x \in S} p_{N}(x)>(2 / \sqrt{e})^{N}$. We prove more.

THEOREM 1. Let $\omega_{N}$ be an $N$-tuple of points on $S$. Then with some numerical constant $c_{1}>0$, the following inequality holds:

$$
\max _{x \in S} p_{N}(x) \geq\left(1+c_{1}\right)(2 / \sqrt{e})^{N} .
$$

This result is in a sense best possible. We prove

THEOREM 2. For each $N$ there exists an $N$-tuple of points $\omega_{N}$ on $S$ such that the inequality

$$
\max _{x \in S} p_{N}(x) \leq\left(1+c_{2}\right)(2 / \sqrt{e})^{N}
$$

holds with some absolute constant $c_{2}>0$.

Note that Theorems 1 and 2 are true for the unit circle $U$ (instead of the unit sphere) with $c_{1}=c_{2}=1$ and the normalizing factor $(2 / \sqrt{e})^{N}$ omitted. For in this case, the product $p_{N}(x)$ is the modulus of a polynomial on $U$ with its zeros on $U$. Theorem 1 and 2 now follow from the fact that the unit root polynomials are "minimal polynomials" for $U$.

In the case of the unit circle the author proved in a previous paper [7] that Theorem 2 is no longer true if the $N$-tuples $\omega_{N}=\left(x_{1}, x_{2}, \ldots, x_{N}\right)$ are sections of a given infinite sequence $\omega=\left(x_{1}, x_{2}, \ldots\right)$. A similar result can be proved for the unit sphere.

THEOREM 3. Let $\omega=\left(x_{1}, x_{2}, \ldots\right)$ be an infinite sequence of points on $S$. Let $A_{N}(\omega)=(\sqrt{e} / 2)^{N} \max _{x \in S} \prod_{j=1}^{N}\left|x-x_{j}\right|$. Then, for some absolute constant $c>0$ and infinitely many values of $N$, the following inequality holds:

$$
A_{N}(\omega) \geq e^{c \sqrt{\log N}}
$$

The lower bound (3) is probably not best possible. As in the case of the unit circle, one might conjecture that (3) holds with $\log N$ instead of $\sqrt{\log N}$.

The problems considered up to this point have a natural counterpart concerning the product of mutual distances between the points of a set. For an $N$-tuple of points $\omega_{N}=\left(x_{1}, x_{2}, \ldots, x_{N}\right)$ on the unit circle it is known that $\prod_{j<k}\left|x_{j}-x_{k}\right| \leq N^{N / 2}$ is true. In the case of the unit sphere we prove a similar upper bound with the exponent $(N / 4)$ instead of $(N / 2)$. 
THEOREM 4. For each $N$-tuple $\omega_{N}=\left(x_{1}, x_{2}, \ldots, x_{N}\right)$ of points on $S$ the following inequality holds:

$$
\prod_{j<k}\left|x_{j}-x_{k}\right| \leq(2 / \sqrt{e})^{N(N-1) / 2} \cdot N^{N / 4+o(N)} .
$$

Again the result of Theorem 4 is best possible, apart from the exact order of the error term $o(N)$ in the exponent. The point set $\omega_{N}$, which will be constructed in order to prove Theorem 2 , also satisfies the relation

$$
\prod_{j<k}\left|x_{j}-x_{k}\right| \geq(2 / \sqrt{e})^{N(N-1) / 2} \cdot N^{N / 4+o(N)} .
$$

\section{Construction of a good point set}

We begin by proving Theorem 2 . It is sufficient to find, for each $N$, an $N$-tuple $\omega_{N}^{0}=\left(x_{1}, \ldots, x_{N}\right)$ on $S$ such that $\max _{x \in S} q\left(x, \omega_{N}^{0}\right) \leq O(1)$ holds for the function $q\left(x, \omega_{N}^{0}\right)$ defined by (2).

Let $N\left(\geq N_{0}\right)$ be given. Set $M=[\sqrt{N}]$. We introduce spherical coordinates $\theta(0 \leq \theta \leq \pi)$ and $\varphi(0 \leq \varphi<2 \pi)$ on $S$. We define a partition of $S$ into spherical zones in the following way.

Choose numbers $\theta_{\mu}, 0=\theta_{0}<\theta<\cdots<\theta_{M-1}<\theta_{M}=\pi$ in such a way that $(N / 2)\left(\cos \theta_{\mu}-\cos \theta_{\mu+1}\right)(\mu=0,1, \ldots, M-1)$ is a positive integer and that the differences $\left(\theta_{\mu+1}-\theta_{\mu}\right)$ are bounded from above and from below by const $/ \sqrt{N}$. Set $N_{\mu}=(N / 2)\left(\cos \theta_{\mu}-\cos \theta_{\mu+1}\right)$. On each of the $M$ circles of latitude $\theta=\xi_{\mu}=\left(\theta_{\mu}+\theta_{\mu+1}\right) / 2(\mu=0,1, \ldots, M-1)$ we place $N_{\mu}$ points; at the vertices of a regular $N_{\mu}$-gon. The position of the regular $N_{\mu}$-gon on the circle is arbitrary. In this way, $\sum_{\mu=0}^{M-1} N_{\mu}=\frac{N}{2} \sum_{\mu=0}^{M-1}\left(\cos \theta_{\mu}-\cos \theta_{\mu+1}\right)=N$ points are distributed over the surface $S$.

Denote the point set defined in this way by $\omega_{N}^{0}$, its points by $x_{\mu \alpha}(\mu=$ $\left.0, \ldots, M-1 ; \alpha=0,1, \ldots, N_{\mu}-1\right)$, and their coordinates by $\left(\xi_{\mu}, \varphi_{\mu \alpha}\right)$ where we choose $\varphi_{\mu \alpha}=2 \pi \alpha / N_{\mu}$ for convenience.

We shall prove

$$
\max _{x \in S} q\left(x, \omega_{N}^{0}\right) \leq O(1) .
$$

As a first step, we split the distance function $\left(x=(\theta, \varphi), x_{0}=\left(\theta_{0}, \varphi_{0}\right)\right)$ $d\left(x, x_{0}\right)=\log \left|x-x_{0}\right|-\log (2 / \sqrt{e})$ into two parts. Let

$$
\begin{array}{r}
e\left(\theta, \theta_{0}\right)=e_{-}\left(\theta, \theta_{0}\right):=\log (e / 4)+\log (1+\cos \theta)+\log \left(1-\cos \theta_{0}\right) \\
\text { for } 0<\theta \leq \theta_{0}<\pi
\end{array}
$$


and

$$
\begin{aligned}
& e\left(\theta, \theta_{0}\right)=e_{+}\left(\theta, \theta_{0}\right):=\log (e / 4)+\log (1-\cos \theta)+\log \left(1+\cos \theta_{0}\right) \\
& \text { for } 0<\theta \leq \theta_{0}<\pi .
\end{aligned}
$$

Furthermore, let

$$
f\left(\theta, \varphi ; \theta_{0}, \varphi_{0}\right)=\log \left|1-\rho e^{i\left(\varphi-\varphi_{0}\right)}\right|
$$

where

$$
\rho=\rho_{-}=\left(\tan \frac{\theta}{2} / \tan \frac{\theta_{0}}{2}\right) \text { for } 0<\theta \leq \theta_{0}<\pi
$$

and

$$
\rho=\rho_{+}=\left(\tan \frac{\theta_{0}}{2} / \tan \frac{\theta}{2}\right) \text { for } 0<\theta_{0} \leq \theta<\pi .
$$

An easy calculation shows that $d\left(x, x_{0}\right)=\frac{1}{2} e\left(\theta, \theta_{0}\right)+f\left(\theta, \varphi ; \theta_{0}, \varphi_{0}\right)$. Given the set $\omega_{N}^{0}$, let $e_{\mu}(\theta)=e\left(\theta, \xi_{\mu}\right)$ and $f_{\mu \alpha}(\theta, \varphi)=f\left(\theta, \varphi ; \xi_{\mu}, \varphi_{\mu \alpha}\right)(\mu=0, \ldots$, $\left.M-1 ; \alpha=0, \ldots, N_{\mu}-1\right)$.

In order to prove (4), it is sufficient to show that the two functions

$$
E(\theta)=\sum_{\mu=0}^{M-1} N_{\mu}-e_{\mu}(\theta), \quad F(\theta, \varphi)=\sum_{\mu, \alpha} f_{\mu \alpha}(\theta, \varphi)
$$

are bounded from above.

LEMMA 1. $E(\theta) \leq O(1)$.

Proof. Let $\theta \in(0, \pi)$. Choose $m \in\{0,1, \ldots, M-1\}$ such that $\xi_{m-1}<$ $\theta \leq \xi_{m}$ holds. The case when $0<\theta<\xi_{0}$ or $\xi_{M-1}<\theta<\pi$ is settled in a similar way. By (5), (7), and the construction of $\omega_{N}^{0}$, we have

$$
\begin{aligned}
E(\theta) & =\sum_{\mu=0}^{M-1} N_{\mu} e\left(\theta, \xi_{\mu}\right)=\frac{N}{2} \sum_{\mu=0}^{M-1}\left(\cos \theta_{\mu}-\cos \theta_{\mu+1}\right) \cdot e\left(\theta, \xi_{\mu}\right) \\
& =\frac{N}{2} \sum_{\mu=0}^{M-1} \Delta \theta_{\mu} \cdot \sin \xi_{\mu} \cdot\left(1+O\left(\left(\Delta \theta_{\mu}\right)^{2}\right)\right) \cdot e\left(\theta, \xi_{\mu}\right) .
\end{aligned}
$$

Here we set $\Delta \theta_{\mu}=\theta_{\mu+1}-\theta_{\mu}$. Using the relation $\Delta \theta_{\mu} \ll N^{-1 / 2}$, we obtain $E(\theta)=\frac{N}{2} \sum_{\mu=0}^{M-1} \Delta \theta_{\mu} \cdot \sin \xi_{\mu} \cdot e\left(\theta, \xi_{\mu}\right)+O\left(\max _{\mu}\left|\sin \xi_{\mu} \cdot e\left(\theta, \xi_{\mu}\right)\right|\right)$. The term $\sin \xi_{\mu} \cdot e\left(\theta, \xi_{\mu}\right)$ is bounded, uniformly in $\xi_{\mu}$ and $\theta$, as can be seen from the definition (5) and the inequality $|t \cdot \log t| \leq e^{-1}$, valid for $0<t \leq 1$. Hence we get

$$
E(\theta)=\frac{N}{2} \sum_{\mu=0}^{M-1} \Delta \theta_{\mu} \cdot \sin \xi_{\mu} \cdot e\left(\theta, \xi_{\mu}\right)+O(1)=E^{*}(\theta)+O(1)
$$


It remains to prove that $E^{*}(\theta)$ is bounded from above. We have

$$
E^{*}(\theta)=\frac{N}{2} \sum_{\mu=0}^{m-1} \Delta \theta_{\mu} \cdot \sin \xi_{\mu} \cdot e_{+}\left(\theta, \xi_{\mu}\right)+\frac{N}{2} \sum_{\mu=m}^{M-1} \Delta \theta_{\mu} \cdot \sin \xi_{\mu} \cdot e_{-}\left(\theta, \xi_{\mu}\right) .
$$

We interpret the first sum as a Riemann sum for the integral $\frac{N}{2} \int_{0}^{\theta_{m}} \sin t$. $e_{+}(\theta, t) d t$ and obtain (see [4], Part II, Problem 11)

$$
\begin{aligned}
& \frac{N}{2} \sum_{\mu=0}^{m-1} \Delta \theta_{\mu} \cdot \sin \theta_{\mu} \cdot e_{+}\left(\theta, \xi_{\mu}\right) \\
& \quad=\frac{N}{2} \int_{0}^{\theta_{m}} \sin t \cdot e_{+}(\theta, t) d t-\frac{N}{48} \sum_{\mu=0}^{m-1}\left(\Delta \theta_{\mu}\right)^{3}\left(\sin t \cdot e_{+}(\theta, t)\right)_{t=\eta_{\mu}}^{\prime \prime}
\end{aligned}
$$

where $\theta_{\mu}<\eta_{\mu}<\theta_{\mu+1}$. A calculation shows that

$$
\begin{aligned}
& -\frac{d^{2}}{d t^{2}}\left(\sin t \cdot e_{+}(\theta, t)\right) \\
& \quad=\sin t\left(\log \frac{e^{2}}{4}+\frac{\cos t}{1+\cos t}+\log (1-\cos \theta)+\log (1+\cos t)\right),
\end{aligned}
$$

and this expression is bounded from above for $\theta, t \in(0, \pi)$. Hence

$$
\frac{N}{2} \sum_{\mu=0}^{m-1} \Delta \theta_{\mu} \cdot \sin \xi_{\mu} \cdot e_{+}\left(\theta, \xi_{\mu}\right) \leq \int_{0}^{\theta_{m}} \sin t \cdot e_{+}(\theta, t) d t+O(1) .
$$

A similar estimate shows that

$$
\frac{N}{2} \sum_{\mu=0}^{M-1} \Delta \theta_{\mu} \cdot \sin \xi_{\mu} \cdot e_{-}\left(\theta, \xi_{\mu}\right) \leq \int_{\theta_{m}}^{\pi} \sin t \cdot e_{-}(\theta, t) d t+O(1) .
$$

Adding (8) and (9), and carrying out the integrations, we obtain

$$
\begin{aligned}
E^{*}(\theta) \leq & O(1)+\frac{N}{2}\left(1-\cos \theta_{m}\right)\left(\log (1-\cos \theta)-\log \left(1-\cos \theta_{m}\right)\right) \\
& +\frac{N}{2}\left(1+\cos \theta_{m}\right)\left(\log (1+\cos \theta)-\log \left(1+\cos \theta_{m}\right)\right) \\
= & O(1)+R\left(\theta, \theta_{m}\right) .
\end{aligned}
$$

The term $R\left(\theta, \theta_{m}\right)$ assumes its maximum value 0 at $\theta=\theta_{m}$, and is negative elsewhere. This proves Lemma 1.

LEMMA 2. $F(\theta, \varphi) \leq O(1)$.

Proof. Let again $\xi_{m-1}<\theta \leq \xi_{m}, 0 \leq \varphi<2 \pi$. Note that the points of $\omega_{N}^{0}$ have coordinates $\left(\xi_{\mu}, \varphi_{\mu \alpha}\right)\left(\mu=0, \ldots, M-1 ; \alpha=0, \ldots, N_{\mu}-1\right)$ where 
$\theta_{\mu \alpha}=2 \pi \alpha / N_{\mu}$. For $\mu \leq m-1$ fixed we have

$$
\begin{aligned}
& \sum_{\alpha=0}^{N_{\mu}-1} f_{\mu \alpha}(\theta, \varphi)=\sum_{\lambda_{\alpha=0}}^{N_{\mu}-1} \log \left|1-\rho_{\mu} \cdot e^{i\left(\varphi-\varphi_{\mu \alpha}\right)}\right| \\
& =\log \left|1-\rho_{\mu}^{N_{\mu}} \cdot e^{i N_{\mu} \varphi}\right|
\end{aligned}
$$

where $\rho_{\mu}=\tan \left(\xi_{\mu} / 2\right) / \tan (\theta / 2)$. Similarly, we get for $\mu \geq m$

$$
\sum_{\alpha=0}^{N_{\mu}-1} f_{\mu \alpha}(\theta, \varphi)=\log \left|1-\rho_{\mu}^{\prime N_{\mu}} \cdot e^{i N_{\mu} \varphi}\right|
$$

where $\rho_{\mu}^{\prime}=\tan (\theta / 2) / \tan \left(\xi_{\mu} / 2\right)$. Relations (10) and (11) imply the following inequality:

$$
\begin{aligned}
F(\theta, \varphi) & \leq \sum_{\mu=0}^{m-1} \log \left(1+\rho_{\mu}^{N_{\mu}}\right)+\sum_{\mu=m}^{M-1} \log \left(1+\rho_{\mu}^{\prime N_{\mu}}\right) \\
& \ll \sum_{\mu=0}^{m-1} \rho_{\mu}^{N_{\mu}}+\sum_{\mu=m}^{M-1} \rho_{\mu}^{\prime N_{\mu}} .
\end{aligned}
$$

We may assume that $0<\theta \leq \pi / 2$. In order to obtain an estimate for the first sum in (12), note that $N_{\mu} \geq K_{1} \cdot \mu$ and $\tan \left(\xi_{\mu} / 2\right) / \tan (\theta / 2) \leq 1-$ $\frac{K_{2}}{\theta \sqrt{N}}(m-\mu-1)$ holds for $\mu=0,1, \ldots, m-1$ and suitably chosen absolute constants $K_{1}>0$ and $K_{2}>0$. In addition, it follows from the assumption $\xi_{m-1}<\theta \leq \xi_{m}$ that $\theta \leq K_{3} m / \sqrt{N}$, and hence $\rho_{\mu} \leq 1-K_{4}(m-\mu-1) / m$ where $K_{4}=K_{2} / K_{3}$. We obtain

$$
\begin{aligned}
\sum_{\mu=0}^{m-1} \rho_{\mu}^{N_{\mu}} & \leq \sum_{\mu=0}^{m-1}\left(1-\frac{K_{4}}{m}(m-\mu-1)\right)^{K_{1} \mu} \leq \sum_{\mu=0}^{m-1} e^{-K \cdot \frac{\mu}{m}(m-\mu-1)} \\
& \leq 2 \sum_{\mu=0}^{\left[\frac{m-1}{2}\right]} e^{-K \cdot \frac{\mu}{m}(m-\mu-1)} \ll 2 \sum_{\mu=0}^{\infty} e^{-\frac{K}{2} \mu} \ll 1 .
\end{aligned}
$$

Proceeding in quite a similar way as before, we get the corresponding estimate for the second sum in (12):

$$
\sum_{\mu=m}^{M-1} \rho_{\mu}^{\prime N_{\mu}} \ll 1 .
$$

From (13) and (14) the assertion follows.

Lemma 1 and 2 together imply the validity of Theorem 2 .

It seems remarkable that from the point of view of uniform distribution the point set $\omega_{N}^{0}$ constructed above is not a very good one. Denoting by $\kappa$ 
an arbitrary spherical cap of $S$, by $F(\kappa)$ its area, and by $Z_{N}(\kappa)$ the number of points in $\kappa$, we certainly have

$$
\sup _{\kappa}\left|Z_{N}(\kappa)-\frac{1}{4 \pi} N \cdot F(\kappa)\right| \gg N^{1 / 2}
$$

It is known, however (see [1]), that there exist point distributions for which the left-hand side of $(15)$ is $\ll N^{1 / 4} \cdot \log ^{1 / 2} N$. It seems that a point set $\omega_{N}$ for which the function $q\left(x, \omega_{N}\right)$ possesses a small maximum, must have properties somewhat distinct from those of a point set for which (15) is small.

As mentioned already, the point set $\omega_{N}^{0}$ can also be used to prove that the upper bound in Theorem 4 is best possible. As the proof is even more computational in nature than that of Theorem 2 , we shall not give all the details and restrict ourselves to a brief sketch.

Let $\omega_{N}^{0}=\left(x_{\mu \alpha}\right)\left(\mu=0, \ldots, M-1 ; \alpha=0, \ldots, N_{\mu}-1\right)$ be the point set contructed above, where $x_{\mu \alpha}=\left(\xi_{\mu}, \varphi_{\mu \alpha}\right)$. It is sufficient to prove that the sum

$$
\sum_{(\mu, \alpha) \neq(\nu, \beta)} \log \left|x_{\mu \alpha}-x_{\nu \beta}\right|-N(N-1) \cdot \log \frac{2}{\sqrt{e}}
$$

is bounded from below by $(N / 2) \cdot \log N+O(N)$. Using the functions $e$ and $f$ defined by (5) and (6), we obtain the following representation of the expression (16):

$$
\begin{aligned}
= & \sum_{\mu=0}^{M-1} \sum_{\alpha \neq \beta} f\left(\xi_{\mu}, \varphi_{\mu \alpha} ; \xi_{\mu}, \varphi_{\mu \beta}\right)+\sum_{\mu \neq \nu} \sum_{\alpha, \beta} f\left(\xi_{\mu}, \varphi_{\mu \alpha} ; \xi_{\nu}, \varphi_{\nu \beta}\right) \\
& +\frac{1}{2} \sum_{\mu \neq \nu} N_{\mu} N_{\nu} e\left(\xi_{\mu}, \xi_{\nu}\right)+\frac{1}{2} \sum_{\mu}\left(N_{\mu}-1\right) e\left(\xi_{\mu}, \xi_{\mu}\right) \\
= & (\mathrm{I})+(\mathrm{II})+(\mathrm{III})+(\mathrm{IV}) .
\end{aligned}
$$

The first sum (I) constitutes the main term and can be evaluated as follows:

$$
\begin{aligned}
(\mathrm{I}) & =\sum_{\mu=0}^{M-1} N_{\mu} \log N_{\mu}=N \log \sqrt{N}+\sum_{\mu=0}^{M-1} N_{\mu} \log \frac{N_{\mu}}{\sqrt{N}} \\
& =\frac{N}{2} \log N+\sqrt{N} \sum_{\mu=0}^{M-1} \frac{N_{\mu}}{\sqrt{N}} \log \frac{N_{\mu}}{\sqrt{N}}=\frac{N}{2} \log N+O(N) .
\end{aligned}
$$

The second sum (II) is bounded by $O(N)$. In order to prove this, we may proceed as in the proof of Lemma 2, noting however that this time we need a lower bound instead of an upper bound. Finally, following the idea of the 
proof of Lemma 1, we can also show that the sums (III) and (IV) are bounded by $O(N)$.

\section{Proof of Theorem 1}

The proof of Theorem 1 is based on the remarkable fact that the distance function $d\left(x, x_{0}\right)=\log \left|x-x_{0}\right|+\log (\sqrt{e} / 2)$ is superharmonic on $S$. As the Laplace operator $\Delta$ is rotation invariant, it is sufficient to consider $\Delta d\left(x, x_{0}\right)$ where $x_{0}$ is the north pole on $S$. In this case,

$$
d\left(x, x_{0}\right)=\log \left(2 \sin \frac{\theta}{2}\right)+\log \frac{\sqrt{e}}{2}
$$

and

$$
\Delta d\left(x, x_{0}\right)=\Delta\left(\log \sin \frac{\theta}{2}\right)=\frac{1}{\sin \theta} \frac{\partial}{\partial \theta}\left(\sin \theta \frac{\partial}{\partial \theta}\left(\log \sin \frac{\theta}{2}\right)\right)=-\frac{1}{2} .
$$

Let $\omega_{N}=\left(x_{1}, x_{2}, \ldots, x_{N}\right)$ be an arbitrary $N$-tuple of points on $S$. It follows from the preceding remark that $\Delta q\left(x, \omega_{N}\right)=-N / 2$ holds for all points $x \neq$ $x_{j}(j=1,2, \ldots, N)$ on $S$.

In accordance with the point set $\omega_{N}$, we construct a test function $T(\theta, \varphi)$ in the following way. Consider the domain $D \subset S$ defined by

$$
D=\left\{(\theta, \varphi): 0 \leq \frac{\pi}{2}=\omega-\theta \leq \frac{\pi}{6}, 0 \leq \varphi \leq \frac{\pi}{2}\right\} .
$$

(The choice of $D$ is rather arbitrary.) Let $r$ be an integer satisfying $2 N \leq$ $4^{r}<8 N$. We decompose the domain $D$ into $4^{r}$ "squares"

$$
\begin{aligned}
B_{\mu \nu}=\left\{(\theta, \varphi): \frac{\pi}{6}(\nu-1) \cdot 2^{-r} \leq \frac{\pi}{2}-\theta \leq\right. & \frac{\pi}{6} \cdot \nu \cdot 2^{-r} ; \\
& \left.\frac{\pi}{6}(\mu-1) \cdot 2^{-r} \leq \varphi \leq \frac{\pi}{6} \mu \cdot 2^{-r}\right\}
\end{aligned}
$$

with $\mu, \nu$ both running from 1 to $2^{r}$. There are two kinds of squares $B_{\mu \nu}$ : squares of the first kind, denoted by $B_{\mu \nu}^{\prime}$, that contain some point $x_{j}$ in their interior, and squares of the second kind, denoted by $B_{\mu \nu}^{\prime \prime}$, that are free from such points. Note that the total area of squares $B_{\mu \nu}^{\prime \prime}$ satisfies the relation $\sum \sigma\left(B_{\mu \nu}^{\prime \prime}\right) \gg 1\left(\sigma=\right.$ area measure). On each $B_{\mu \nu}$ we define a function $\tau_{\mu \nu}(\theta, \varphi)$ as follows. If $B_{\mu \nu}$ is of the first kind, let $\tau_{\mu \nu}(\theta, \varphi) \equiv 0$. If $B_{\mu \nu}$ is of the second kind, let $\tau_{\mu \nu}(\theta, \varphi)=\sin ^{2}\left(6 \cdot 2^{r} \theta\right) \cdot \sin ^{2}\left(6 \cdot 2^{r} \varphi\right)$. Note that the normal derivative of $\tau_{\mu \nu}$ with respect to the boundary of $B_{\mu \nu}$ vanishes, and that

$$
\begin{aligned}
& \left|\Delta \tau_{\mu \nu}(\theta, \varphi)\right|=\left|\sin ^{-2} \theta \cdot \frac{\partial^{2} \tau_{\mu \nu}}{\partial \varphi^{2}}+\sin ^{-1} \theta \cdot \frac{\partial}{\partial \theta}\left(\sin \theta \cdot \frac{\partial \tau_{\mu \nu}}{\partial \theta}\right)\right| \\
& \ll 4^{r} \ll N
\end{aligned}
$$


holds everywhere. Now define the test function $T(\theta, \varphi)$ on $S$ by $T(\theta, \varphi)=$ $\Delta \tau_{\mu \nu}(\theta, \varphi)$ for $(\theta, \varphi) \in B_{\mu \nu}$, and $T(\theta, \varphi)=0$ for $(\theta, \varphi) \notin D$. Using Green's second formula for domains on curved surfaces (see, for example, [2, Section 91]), we obtain the following estimate:

$$
\begin{aligned}
\int_{S}\left|q_{N}(x)\right| d \sigma(x) \cdot \sup _{x \in S}|T(x)| \geq\left|\int_{S} q_{N}(x) T(x) d \sigma(x)\right| \\
=\left|\sum_{\mu, \nu} \int_{B_{\mu \nu}} q_{N}(x) \cdot \Delta \tau_{\mu \nu}(x) d \sigma(x)\right|=\left|\sum_{\mu, \nu} \int_{B_{\mu \nu}} \Delta q_{N}(x) \tau_{\mu \nu}(x) d \sigma(x)\right| \\
=\frac{N}{2} \sum_{\mu, \nu} \int_{B_{\mu \nu}^{\prime \prime}} \tau_{\mu \nu}(x) d \sigma(x) \gg \frac{N}{2} \sum \sigma\left(B_{\mu \nu}^{\prime \prime}\right) \gg N .
\end{aligned}
$$

Recalling inequality (17), we find that $\sup _{x \in S}|T(x)| \ll N$ holds, and that finally

$$
\int_{S}\left|q_{N}(x)\right| d \sigma(x) \gg 1 .
$$

In view of the relation $\int_{S} q_{N}(x) d \sigma(x)=0,(18)$ is even stronger than the assertion of Theorem 1. This finishes the proof of Theorem 1.

\section{Proof of Theorem 3}

In order to prove Theorem 3 we make use of two ideas. The first one is due to K. F. Roth [6] and consists in replacing the dynamic problem by a static one. The second idea is due to G. Halasz [3]: different test functions are combined in the form of a Riesz product to obtain a lower bound for the maximum in question.

Let $\omega=\left(x_{1}, x_{2}, \ldots\right)$ be an infinite sequence on $S$. Define numbers $a_{n}(\omega)$ by

$$
a_{n}(\omega)=\max _{x \in S}\left(\sum_{j=1}^{n} \log \left|x-x_{j}\right|+\log \frac{\sqrt{e}}{2}\right) .
$$

Consider the section $\omega_{N}=\left(x_{1}, x_{2}, \ldots, x_{N}\right)$ of $\omega$ with $N=4^{l^{2}}$ where $l$ is a fixed positive integer. We shall prove that

$$
\max _{1 \leq n \leq N} a_{n}(\omega) \gg l
$$

from which the assertion follows. Denote by $X$ the box $S \times[0,1)=\{(\theta, \varphi, t)$ : $0<\theta<\pi, 0 \leq \varphi<2 \pi, 0 \leq t<1\}$. Let $X$ be endowed with the product measure $v=\sigma \times \lambda$, where $\sigma$ is the usual area measure on $S$, and $\lambda$ is the onedimensional Lebesgue measure. To each point $x_{j}=\left(\theta_{j}, \varphi_{j}\right)$ of the section 
$\omega_{N}$, we assign as a counterpart on $X$ the point $y_{j}=\left(\theta_{j}, \varphi_{j},(j-1) / N\right)(j=$ $1,2, \ldots, N)$. Define a function $Q(y)=Q(\theta, \varphi, t)$ on $X$ by

$$
Q(\theta, \varphi, t)=q_{[N t]+1}(x)=\sum_{j=1}^{[N t]+1}\left(\log \left|x-x_{j}\right|+\log \frac{\sqrt{e}}{2}\right) .
$$

Following Roth's idea, it is sufficient to prove the lower estimate

$$
\max _{X} Q(\theta, \varphi, t) \gg l,
$$

from which (19) follows. In the sequel, we may assume without loss of generality that $\int_{X}|Q| d v \ll l$, otherwise the assertion follows in view of the relation $\int_{X} Q d v=0$.

Now we use the method of Halasz. We construct a test function $T(y)=$ $\prod_{\alpha=1}^{l}\left(1+\rho R_{\alpha}(y)\right)$ on $X$, where $0<\rho<1 / 2$ is a suitably chosen parameter, and the functions $R_{\alpha}(y)$ possess the following properties:

and

(1) $\left|R_{\alpha}(y)\right| \leq 1$ for $y \in X$ and $\alpha=1,2, \ldots, l$;

(2) $\int_{X} R_{\alpha_{1}}(y) \cdot R_{\alpha_{2}}(y) \cdots R_{\alpha_{s}}(y) d v(y)=0$ for $1 \leq \alpha_{1}<\alpha_{2}<\cdots<\alpha_{s} \leq l$. These two properties imply that $\int_{X} T(y) d v(y)=\int_{X} 1 \cdot d v(y)=4 \pi$ holds. We have

$$
\max _{y \in X} Q(y)=\frac{1}{4 \pi} \max _{y \in X} Q(y) \cdot \int_{X} T(y) d v(y) \geq \frac{1}{4 \pi} \int_{X} Q(y) T(y) d v(y) .
$$

The functions $R_{\alpha}(y)$ are chosen in such a way that the linear terms $\rho \cdot R_{\alpha}$ in the expansion of $\prod_{\alpha=1}^{l}\left(1+\rho R_{\alpha}(y)\right)$ give the main contribution to the integral on the right of (20), whereas the mixed terms $\rho^{s} R_{\alpha_{1}} R_{\alpha_{2}} \cdots R_{\alpha_{s}}(s \geq 2)$ produce error terms dominated by the main contribution.

Let $X^{*} \subset X$ be the subdomain $X^{*}=D \times[0,1)$, where $D$ is the domain introduced in Section 3. Let $\alpha \in\{1,2, \ldots, l\}$ and define $n_{\alpha}=2^{\alpha^{2}}$. Note that $N=4^{l^{2}}=n_{l}^{2}$. Let $P_{\alpha}$ be a partition of $X^{*}$ into boxes $A_{\mu \nu, \lambda}^{(\alpha)}$, where $A_{\mu \nu, \lambda}^{(\alpha)}$ is the cartesian product of the box

$$
\begin{aligned}
B_{\mu \nu}^{(\alpha)}=\left\{(\theta, \varphi): \frac{\pi}{6} \cdot(\nu-1) n_{\alpha}^{-1} \leq\right. & \theta \\
& \leq \frac{\pi}{6} \cdot \nu \cdot n_{\alpha}^{-1} ; \\
& \left.\frac{\pi}{6} \cdot(\mu-1) n_{\alpha}^{-1} \leq \varphi \leq \frac{\pi}{6} \cdot \mu \cdot n_{\alpha}^{-1}\right\} \subset D
\end{aligned}
$$

with the interval $C_{\lambda}^{(\alpha)}=\left\{t:(\lambda-1) \cdot \frac{n_{\alpha}^{2}}{2 N} \leq t \leq \lambda \cdot \frac{n_{\alpha}^{2}}{2 N}\right\}$. The indices $\mu$ and $\nu$ run from 1 to $n_{\alpha}$, the index $\lambda$ runs from 1 to $2 N / n_{\alpha}^{2}$. Hence, for each $\alpha, 1 \leq \alpha \leq l$, we have a partition of $X^{*}$ into $2 N$ boxes $A_{\mu \nu, \lambda}^{(\alpha)}$. Again, there exist two kinds of boxes: boxes of the first kind that contain some point $y_{j}$ as an interior point, and boxes of the second kind that are free from such points. 

by

On each box of the second kind we define a function $u_{\mu \nu, \lambda}^{(\alpha)}(y)=u_{\mu \nu, \lambda}^{(\alpha)}(\theta, \varphi, t)$

$$
u_{\mu \nu, \lambda}^{(\alpha)}(\theta, \varphi, t)=\Delta \tau_{\mu \nu}(\theta, \varphi) \cdot \operatorname{sign} t
$$

where sign $=-1$ for $(\lambda-1) \frac{n_{\alpha}^{2}}{2 N} \leq t<\left(\lambda-\frac{1}{2}\right) \frac{n_{\alpha}^{2}}{2 N}, \operatorname{sign} t=+1$ for $\left(\lambda-\frac{1}{2}\right) \frac{n_{\alpha}^{2}}{2 N} \leq$ $t<\lambda \cdot \frac{n_{\alpha}^{2}}{2 N}$, and $\tau_{\mu \nu}(\theta, \varphi)=-c \cdot n_{\alpha}^{-2} \cdot \sin ^{2} 6 n_{\alpha} \theta \cdot \sin ^{2} 6 n_{\alpha} \varphi$. Here $c>0$ is an absolute constant ( $c=1 / 1000$ will do) chosen in such a way that the spherical Laplacian derivative $\Delta \tau_{\mu \nu}(\theta, \varphi)$ is bounded by 1 in absolute value.

On boxes of the first kind, set $u_{\mu \nu, \lambda}^{(\alpha)}(y)=0$. Finally, define $R_{\alpha}(y)=u_{\mu \nu, \lambda}^{(\alpha)}(y)$ for $y \in A_{\mu \nu, \lambda}^{(\alpha)}, R_{\alpha}(y)=0$ for $y \in X \backslash X^{*}$, and let $T(y)=\prod_{\alpha=1}^{l}\left(1+\rho \cdot R_{\alpha}(y)\right)$. The parameter $\rho, 0<\rho<1 / 2$, will be chosen later on.

First of all, we shall prove that the $R_{\alpha}$ 's form a system of functions completely orthogonal with respect to the measure $v$.

LemMa 3. Let $1 \leq \alpha_{1}<\alpha_{2}<\cdots<\alpha_{s} \leq l$. Then the following orthogonality relation holds: $\int_{X} R_{\alpha_{1}}(y) R_{\alpha_{2}}(y) \cdots R_{\alpha_{s}}(y) d v(y)=0$.

Proof. For fixed $\theta, \varphi$ consider the set $\left\{(\theta, \varphi, t):(\lambda-1) n_{\alpha_{1}}^{2} / 2 N \leq t<\right.$ $\left.\lambda n_{\alpha_{1}}^{2} / 2 N\right\}$. The functions $R_{\alpha_{2}}, \ldots, R_{\alpha_{s}}$ are of constant value on this interval, whereas $R_{\alpha_{1}}$ is of constant absolute value but changes sign in the middle of the interval. Integrating first with respect to $d t$, then with respect to $\sin \theta d \theta d \varphi$, proves the assertion.

COROLLARY 1. $\int_{X} T(y) d v(y)=\int_{X} d v(y)=4 \pi$.

LEMMA 4. For each box $A_{\mu \nu, \lambda}^{(\alpha)}$ of the second kind, the following inequality holds:

$$
\int_{A_{\mu \nu, \lambda}^{(\alpha)}} Q(y) R_{\alpha}(y) d v(y) \gg N^{-1}
$$

Proof. Fix $t_{0},(\lambda-1) n_{\alpha}^{2} / 2 N \leq t_{0}<\left(\lambda-\frac{1}{2}\right) n_{\alpha}^{2} / 2 N$, and set $t_{1}=t_{0}+\frac{1}{2} n_{\alpha}^{2} / 2 N$. Note that $R_{\alpha}\left(\theta, \varphi, t_{0}\right)=-R_{\alpha}\left(\theta, \varphi, t_{1}\right)$. We obtain the integral in question by first integrating $Q\left(\theta, \varphi, t_{0}\right) \cdot R_{\alpha}\left(\theta, \varphi, t_{0}\right)+Q\left(\theta, \varphi, t_{1}\right) \cdot R_{\alpha}\left(\theta, \varphi, t_{1}\right)$ with respect to $d \sigma(\theta, \varphi)$ over $B_{\mu \nu}^{(\alpha)}$, using Green's formula as in the proof of Theorem 1, and then with respect to $d t_{1}$ over the interval $\left(\lambda-\frac{1}{2}\right) n_{\alpha}^{2} / 2 N \leq t_{1} \leq \lambda n_{\alpha}^{2} / 2 N$. 
We have

$$
\begin{aligned}
& \int_{B_{\mu \nu}^{(\alpha)}}\left(Q\left(\theta, \varphi, t_{0}\right) \cdot R_{\alpha}\left(\theta, \varphi, t_{0}\right)+Q\left(\theta, \varphi, t_{1}\right) \cdot R_{\alpha}\left(\theta, \varphi, t_{1}\right)\right) d \sigma \\
& =\int_{B_{\mu \nu}^{(\alpha)}} R_{\alpha}\left(\theta, \varphi, t_{1}\right)\left(Q\left(\theta, \varphi, t_{1}\right)-Q\left(\theta, \varphi, t_{0}\right)\right) d \sigma \\
& =\int_{B_{\mu \nu}^{(\alpha)}} \Delta \tau_{\mu \nu}^{(\alpha)}(\theta, \varphi) \cdot \sum_{\left[N t_{0}\right]+2}^{\left[N t_{1}\right]+1}\left(\log \left|x-x_{j}\right|+\log \frac{\sqrt{e}}{2}\right) d \sigma(\theta, \varphi) \\
& \gg N\left(t_{1}-t_{0}\right) \cdot \sigma\left(B_{\mu \nu}^{(\alpha)}\right) \cdot n_{\alpha}^{-2} \gg n_{\alpha}^{2} \cdot \sigma\left(B_{\mu \nu}^{(\alpha)}\right) \cdot n_{\alpha}^{-2}=\sigma\left(B_{\mu \nu}^{(\alpha)}\right)
\end{aligned}
$$

Integrating (22) with respect to $d t_{1}$, and noting that $v\left(A_{\mu \nu, \lambda}^{(\alpha)}\right) \gg N^{-1}$, we obtain the desired result. This proves Lemma 4.

Summing (21) over all boxes $A_{\mu \nu \lambda}^{(\alpha)}$ of the second kind, we get

COROLlaRY 2. $\sum_{\alpha=1}^{l} \rho \int_{X} Q(y) R_{\alpha}(y) d v(y) \gg \rho \cdot l$.

In a final step we show that the remaining terms in the expansion of $\prod_{\alpha=1}^{l}\left(1+\rho R_{\alpha}(y)\right)$ give a contribution which is dominated by $\rho \cdot l$.

LEMMA 5. The following inequality holds:

$$
\left|\sum_{\alpha_{1}<\alpha_{2}} \rho^{2} \int_{X} Q R_{\alpha_{1}} R_{\alpha_{2}} d v+\sum_{\alpha_{1}<\alpha_{2}<\alpha_{3}} \rho^{3} \int_{X} Q R_{\alpha_{1}} R_{\alpha_{2}} R_{\alpha_{3}} d v+\cdots\right| \ll \rho^{2} \cdot l .
$$

Proof. Let $1 \leq \alpha_{1}<\alpha_{2}<\cdots<\alpha_{s} \leq l$. Consider a box of the form $B_{\mu \nu}^{\left(\alpha_{s}\right)} \times C_{\lambda}^{\left(\alpha_{1}\right)}$. Denote by $I_{1}, I_{2}$ the two halves of the interval $C_{\lambda}^{\left(\alpha_{1}\right)}$. For fixed $\theta, \varphi$, the functions $R_{\alpha_{2}}, \ldots, R_{\alpha_{s}}$ are constant in the variable $t$ on $B_{\mu \nu}^{\left(\alpha_{s}\right)} \times C_{\lambda}^{\left(\alpha_{1}\right)}$, whereas $R_{\alpha_{1}}$ is constant in absolute value, but changes sign in the middle of $C_{\lambda}^{\left(\alpha_{1}\right)}$. We split the product $R_{\alpha_{1}}(y) \cdot R_{\alpha_{2}}(y) \cdots R_{\alpha_{s-1}}(y)$ on $B_{\mu \nu}^{\left(\alpha_{s}\right)} \times I_{2}$ into two parts: $R_{\alpha_{1}}(y) \cdots \cdots R_{\alpha_{s-1}}(y)=m+\varepsilon(y)$, where $m$ is the mean value on $B_{\mu \nu}^{\left(\alpha_{s}\right)} \times I_{2}$ with respect to $v$, and $\varepsilon(y)$ is the error term. Similarly, on $B_{\mu \nu}^{\left(\alpha_{s}\right)} \times I_{1}$ we get $R_{\alpha_{1}}(y) \cdots \cdot R_{\alpha_{s-1}}(y)=-m+\varepsilon(y)$. In view of $\left|R_{\alpha}(y)\right| \leq 1$, we have the inequalities $|m| \leq 1$ and

$$
|\varepsilon(y)| \leq \frac{\pi}{6} \cdot n_{\alpha_{s}}^{-1} \cdot \sup _{X}\left(\left|\frac{\partial}{\partial \theta} R_{\alpha_{1}} \cdots R_{\alpha_{s-1}}\right|+\left|\frac{\partial}{\partial \varphi} R_{\alpha_{1}} \cdots R_{\alpha_{s-1}}\right|\right) \ll n_{\alpha_{s-1}} / n_{\alpha_{s}} .
$$


Integrating over $B_{\mu \nu}^{\left(\alpha_{s}\right)} \times C_{\lambda}^{\left(\alpha_{1}\right)}=B_{\mu \nu}^{\left(\alpha_{1}\right)}=B_{\mu \nu}^{\left(\alpha_{s}\right)} \times\left(I_{1} \cup I_{2}\right)$, we obtain the following inequality:

$$
\begin{aligned}
& \left|\int_{B_{\mu \nu}^{\left(\alpha_{\nu}\right)} \times C_{\lambda}^{\left(\alpha_{1}\right)}} Q(y) R_{\alpha_{1}}(y) \cdots R_{\alpha_{s}}(y) d v(y)\right| \\
& =\mid \int_{B_{\mu \nu}^{\left(s_{s}\right)} \times I_{1}}(-m+\varepsilon(y)) Q(y) R_{\alpha_{s}}(y) d v(y) \\
& \quad+\int_{B_{\mu \nu}^{\left(\alpha_{s}\right)} \times I_{2}}(m+\varepsilon(y)) Q(y) R_{\alpha_{s}}(y) d v(y) \mid \\
& \quad \ll\left|\int_{B_{\mu \nu}^{\left(s_{s}\right)} \times I_{2}} Q(y) R_{\alpha_{s}}(y) d v(y)-\int_{B_{\mu \nu}^{\left(\alpha_{s}\right)} \times I_{1}} Q(y) R_{\alpha_{s}}(y) d v(y)\right| \\
& \quad+\left(n_{\alpha_{s-1}} / n_{\alpha_{s}}\right) \cdot \int_{B_{\mu \nu}^{\left(\alpha_{s}\right)} \times C_{\lambda}^{\left(\alpha_{1}\right)}}|Q(y)| d v(y) \\
& \ll\left(n_{\alpha_{1} / n_{\alpha_{s}}}\right)^{2} \cdot v\left(B_{\mu \nu}^{\left(\alpha_{s}\right)} \times C_{\lambda}^{\left(\alpha_{1}\right)}\right)+\left(n_{\alpha_{n-1}} / n_{\alpha_{s}}\right) \cdot \int_{B_{\mu \nu}^{\left(\alpha_{s}\right)} \times C_{\lambda}^{\left(\alpha_{1}\right)}}|Q(y)| d v(y) .
\end{aligned}
$$

In order to obtain the first term in (23), we may proceed as in the proof of Lemma 4 , replacing the symbol $\gg$ by $\ll$, and noting that $N\left(t_{1}-t_{0}\right) \ll n_{\alpha_{1}}^{2}$ in our case.

Summing (23) over all boxes $B_{\mu \nu}^{\left(\alpha_{s}\right)} \times C_{\lambda}^{\left(\alpha_{1}\right)}$, and noting that $\int_{X}|Q(y)| d y(y) \ll$ $l$ without loss of generality, we get

$$
\begin{array}{rl}
\mid \int_{X} & Q(y) R_{\alpha_{1}}(y) \cdots \cdots R_{\alpha_{s}}(y) d v(y) \mid \\
& \left.\ll n_{\alpha_{1}}^{2} / n_{\alpha_{s}}^{2}\right)+l \cdot\left(n_{\alpha_{s-1}} / n_{\alpha_{s}}\right) \\
& \ll l \cdot\left(n_{\alpha_{s-1}} / n_{\alpha_{s}}\right) .
\end{array}
$$

Summing (24) over all $s$-tuples $1 \leq \alpha_{1}<\alpha_{2}<\cdots<\alpha_{s} \leq l$ with $s \geq 2$, we get

$$
\begin{aligned}
& \left|\sum_{\alpha_{1}<\alpha_{2}} \rho^{2} \int_{X} Q R_{\alpha_{1}} R_{\alpha_{2}} d v+\sum_{\alpha_{1}<\alpha_{2}<\alpha_{3}} \rho^{3} \int_{X} Q R_{\alpha_{1}} R_{\alpha_{2}} R_{\alpha_{3}} d v+\cdots\right| \\
& \quad \ll \rho^{2} l \sum_{\alpha_{1}<\alpha_{2}}\left(n_{\alpha_{1}} / n_{\alpha_{2}}\right)+\rho^{3} l \sum_{\alpha_{1}<\alpha_{2}<\alpha_{3}}\left(n_{\alpha_{2}} / n_{\alpha_{3}}\right)+\cdots \\
& \quad \ll \rho^{2} l \sum_{\alpha_{1}<\alpha_{2}} 2^{-\alpha_{2}}+j \rho^{3} l \sum_{\alpha_{1}<\alpha_{2}<\alpha_{3}} 2^{-\alpha_{3}}+\cdots \\
& \quad=\rho^{2} l\left(\sum_{\alpha=2}^{l}\left(\begin{array}{c}
\alpha-1 \\
1
\end{array}\right) 2^{-\alpha}+\rho \sum_{\alpha=3}^{l}\left(\begin{array}{c}
\alpha-1 \\
2
\end{array}\right) 2^{-\alpha}+\cdots\right) \\
& \quad=\rho \cdot l \sum_{\alpha=2}^{l} 2^{-\alpha}\left((1+\rho)^{\alpha-1}-1\right) \leq \rho^{2} \cdot l \sum_{\alpha=2}^{l}(\alpha-1)(1+\rho)^{\alpha-2} 2^{-\alpha} \ll \rho^{2} l .
\end{aligned}
$$

This proves Lemma 5 . 
Corollary 2 and Lemma 5 imply the following final inequality:

$$
\begin{aligned}
\int_{X} Q(y) T(y) d v(y)= & \int_{X} Q(y) d v(y)+\rho^{l} \sum_{\alpha=1}^{l} \int_{X} R_{\alpha}(y) Q(y) d v(y) \\
& +\sum_{s=2}^{l} \rho^{s} \sum_{\alpha_{1}<\cdots<\alpha_{s}} \int_{X} Q(y) R_{\alpha_{1}}(y) \cdots R_{\alpha_{s}}(y) d v(y) \\
\geq & c_{1} \rho l-c_{2} \rho^{2} l,
\end{aligned}
$$

where $c_{1}$ and $c_{2}$ are positive absolute constants. Choosing $\rho<c_{1} / 2 c_{2}$, the assertion of Theorem 3 follows in view of relation (20).

\section{On the product of mutual distances}

Let $\omega_{N}=\left(x_{1}, x_{2}, \ldots, x_{N}\right)$ be a point set on $S$, and let $\theta_{j}, \varphi_{j}$ be the spherical coordinates of the points $x_{j}$. We shall derive an upper bound for the product of mutual distances between the points of $\omega_{N}$. The first proof is more elegant and gives a slightly better remainder term, whereas the idea of the second proof is more general and applicable both to higher dimensions and various types of similar kernels.

FIRST PROOF. Set

$$
a_{j}=\cos \left(\theta_{j} / 2\right) \cdot \exp \left(i \varphi_{j} / 2\right) \text { and } b_{j}=\sin \left(\theta_{j} / 2\right) \cdot \exp \left(-i \varphi_{j} / 2\right),
$$

and note that

$$
\left|x_{j}-x_{k}\right|=2 \cdot\left|a_{j} b_{k}-a_{k} b_{j}\right| .
$$

Relation (25) allows us to express the product of mutual distances by means of a determinant of the Vandermonde type. Consider the $(N, N)$-matrix $P=\left(p_{\mu \nu}\right)(\mu, \nu=1,2, \ldots, N)$ where $p_{\mu \nu}=a_{\mu}^{N-\nu} \cdot b_{\mu}^{\nu-1}$. It is easy to prove that

$$
\prod_{j<k}\left|x_{j}-x_{k}\right|=2^{N(N-1) / 2} \cdot|\operatorname{Det} P| .
$$

Now replace the matrix $P$ by a new matrix $P^{\prime}=\left(p_{\mu \nu}^{\prime}\right)$ where

$$
p_{\mu \nu}^{\prime}=p_{\mu \nu} \cdot\left(\begin{array}{c}
N-1 \\
\nu-1
\end{array}\right)^{1 / 2} \quad(\mu, \nu=1,2, \ldots, N) .
$$

Note that $|\operatorname{Det} P|=\left|\operatorname{Det} P^{\prime}\right| \cdot \prod_{\nu=1}^{N}\left(\begin{array}{c}N-1 \\ \nu-1\end{array}\right)^{-1 / 2}$ from which

$$
\prod_{j<k}\left|x_{j}-x_{k}\right|=2^{N(N-1) / 2} \prod_{\nu=1}^{N}\left(\begin{array}{c}
N-1 \\
\nu-1
\end{array}\right)^{-1 / 2} \cdot\left|\operatorname{Det} P^{\prime}\right|
$$


follows. We obtain an upper bound for $\mid$ Det $P^{\prime} \mid$ by applying Hadamard's inequality. The length of the $\mu$ th row vector of $P^{\prime}$ is equal to

$$
\begin{aligned}
\left(\sum_{\nu=1}^{N}\left|p_{\mu \nu}^{\prime 2}\right|\right)^{1 / 2} & =\left(\sum_{\nu=1}^{N}\left(\begin{array}{c}
N-1 \\
\nu-1
\end{array}\right)\left|a_{\mu}^{2}\right|^{N-\nu}\left|b_{\mu}^{2}\right|^{\nu-1}\right)^{1 / 2}=\left(\left|a_{\mu}^{2}\right|+\left|b_{\mu}^{2}\right|\right)^{(N-1) / 2} \\
& =\left(\sin ^{2}\left(\theta_{\mu} / 2\right)+\cos ^{2}\left(\theta_{\mu} / 2\right)\right)^{(N-1) / 2}=1 .
\end{aligned}
$$

Hence $\left|\operatorname{Det} P^{\prime}\right| \leq 1$ and

$$
\prod_{j<k}\left|x_{j}-x_{k}\right| \leq 2^{N(N-1) / 2} \prod_{\nu=1}^{N}\left(\begin{array}{c}
N-1 \\
\nu-1
\end{array}\right)^{-1 / 2} .
$$

It suffices to evaluate $\prod_{\nu=1}^{k}\left(\begin{array}{c}N-1 \\ \nu-1\end{array}\right)=\prod_{\nu=1}^{N-1}\left(\nu^{\nu} / \nu\right.$ !). Using Stirling's formula, we get

$$
\prod_{\nu=1}^{N-1}\left(\nu^{\nu} / \nu !\right)=e^{(1 / 2) N(N-1)} \cdot N^{-(N / 2)+o(N)} .
$$

From (26) and (27) the upper estimate

$$
\prod_{j<k}\left|x_{j}-x_{k}\right| \leq(2 / \sqrt{e})^{N(N-1) / 2} \cdot N^{N / 4+o(N)}
$$

follows.

SECOND PROOF. Let $x$ and $y$ be points on $S$, and let $y$ be the angle between the radius vectors pointing from the center of the sphere to $x$ and $y$, respectively. We have $\log |x-y|=(1 / 2) \log (2-2 \cos \gamma)$. Define a new distance function $d_{r}(x, y)$ on $S$ by setting $d_{r}(x, y)=(1 / 2) \log \left(1+r^{2}-2 r \cos \gamma\right)$. The parameter $r \in(0,1)$ will be chosen later on. Note that the mean value $m_{r}$ of $d_{r}(x, y)$ on $S$ is

$$
\begin{aligned}
m_{r} & =\frac{1}{2} \int_{0}^{\pi} \frac{1}{2} \log \left(1+r^{2}-2 r \cos \theta\right) \sin \theta d \theta \\
& =-\frac{1}{2}+\frac{(1+r)}{4 r} \log (1+r)-\frac{(1-r)^{2}}{4 r} \log (1-r) .
\end{aligned}
$$

For short, let $D(x, y)=\log |x-y|-\log (2 / \sqrt{e})$ and $D_{r}(x, y)=d_{r}(x, y)-m_{r}$. Consider the following trivial inequality:

$$
\begin{aligned}
\sum_{j \neq k} \log \left|x_{j}-x_{k}\right|-N(N-1) \log (2 / \sqrt{e})=\sum_{j \neq k} D\left(x_{j}, x_{k}\right) \\
=\sum_{j, k} D_{r}\left(x_{j}, x_{k}\right)-\sum_{j} D_{r}\left(x_{j}, x_{j}\right)+\sum_{j \neq k}\left(D\left(x_{j}, x_{k}\right)-D_{r}\left(x_{j}, x_{k}\right)\right) \\
\leq \sum_{j, k} D_{r}\left(x_{j}, x_{k}\right)-N \log (1-r)+N \cdot m_{r} \\
\quad+N(N-1) \max _{x, y \in S}\left(D(x, y)-D_{r}(x, y)\right) .
\end{aligned}
$$


A calculation shows that

$$
\max _{x, y \in S}\left(D(x, y)-D_{r}(x, y)\right)=\left((1-r)^{2} / 4 r\right) \log ((1+r) /(1-r)) .
$$

Now choose $r=1-1 / \sqrt{N \log N}$. We obtain

$$
\begin{aligned}
& \sum_{j \neq k} \log \left|x_{j}-x_{k}\right|-N(N-1) \log (2 / \sqrt{e}) \\
& \leq \sum_{j, i k} D_{r}\left(x_{j}, x_{k}\right)+\frac{N}{2} \log N+O(N \log \log N)
\end{aligned}
$$

We shall prove that the first term on the right of (28) is negative. Let $\sum_{n=1}^{\infty} a_{n}(r) P_{n}(\cos \theta)$ be the expansion into spherical harmonics of the kernel $k_{r}(\theta)=(1 / 2) \log \left(1+r^{2}-2 r \cos \theta\right)-m_{r}(0<r<1)$. It is known that all the coefficients $a_{n}(r)$ are negative (the proof works in exactly the same manner as the proof of [5, Hilfssatz 6 , page 36]). Consider the new kernel $\kappa_{r}(\theta)=\sum_{n=1}^{\infty}\left(-a_{n}(r) \cdot \frac{2 n+1}{2 \pi}\right)^{1 / 2} P_{n}(\cos \theta)$, which is a convolution root of $-k_{r}(\theta)$. Let $\delta_{r}(x, y)$ be the distance function generated by $\kappa_{r}(\theta)$ on $S$. Then Legendre's addition formula for spherical harmonics implies the following relation:

$$
\sum_{j, k} D_{r}\left(x_{j}, x_{k}\right)=-\int_{S}\left(\sum_{j} \delta_{r}\left(x, x_{j}\right)\right)^{2} d \sigma(x) \leq 0 .
$$

From (28) and (29) the assertion of Theorem 4 follows a second time.

\section{References}

[1] J. Beck, 'Some upper bounds in the theory of irregularities of distribution', Acta Arith. 43 (1984), 115-130.

[2] W. Blaschke and K. Leichtweiß, Elementare Differentialgeometrie (Springer-Verlag, Berlin, 1973).

[3] G. Halasz, 'On Roth's method in the theory of irregularities of point distributions', Recent progress in analytic number theory, Vol. 2, pp. 79-94 (Durham 1979, Academic Press, 1981).

[4] G. Pólya and G. Szegö, Problems and Theorems in Analysis, Vol. 1 (Springer-Verlag, Berlin, New York, 1972-76).

[5] G. Pólya and G. Szegö, 'On the transfinite diameter (capacity constant) of subsets in the plane and in space', J. Reine Angew. Math. 165 (1931), 4-49. (German) 
[6] K. F. Roth, 'On irregularities of distribution', Mathematika 7 (1954), 73-79.

[7] G. Wagner, 'On a problem of Erdös in diophantine approximation', Bull. London Math. Soc. 12 (1980), 81-88.

Mathematisches Institut A

Pfaffenwaldring 57

D-7000 Stuttgart 80

Federal Republic of Germany 